\title{
Commission 12: Solar Radiation \& Structure
}

\author{
PRESIDENT: Thomas. J. Bogdan \\ VICE-PRESIDENT: Valentin Martínez Pillet \\ ORGANIZING COMMITTEE: M. Asplund, \\ J. Christensen-Dalsgaard, G. Cauzzi, L. E. Cram, D. Dravins, \\ W. Gan, P. Henzl, A. Kosovichev, J. T. Mariska, M. G. Rovira, \\ and P. Venkatakrishnan
}

\begin{abstract}
Commission 12 covers research on the internal structure and dynamics of the Sun, the "quiet" solar atmosphere, solar radiation and its variability, and the nature of relatively stable magnetic structures like sunspots, faculae and the magnetic network. There is considerable productive overlap with the other Commissions of Division II as investigations move progressively toward the fertile intellectual boundaries between traditional research disciplines. In large part, the solar magnetic field provides the linkage that connects these diverse themes. The same magnetic field that produces the more subtle variations of solar structure and radiative output over the $11 \mathrm{yr}$ activity cycle is also implicated in rapid and often violent phenomena such as flares, coronal mass ejections, prominence eruptions, and episodes of sporadic magnetic reconnection.

The last three years have again brought significant progress in nearly all the research endeavors touched upon by the interests of Commission 12. The underlying causes for this success remain the same: sustained advances in computing capabilities coupled with diverse observations with increasing levels of spatial, temporal and spectral resolution. It is all but impossible to deal with these many advances here in anything except a cursory and selective fashion. Thankfully, the Living Reviews in Solar Physics has published several extensive reviews over the last two years that deal explicitly with issues relevant to the purview of Commission 12 . The reader who is eager for a deeper and more complete understanding of some of these advances is directed to http://www.livingreviews.org for access to these articles.
\end{abstract}

Keywords. Sun: abundances, Sun: atmosphere, Sun: chromosphere, Sun: corona, Sun: evolution, Sun: hleioseismology, Sun: magnetic fields, Sun: oscillations, (Sun:) solar wind, (Sun:) sunspots

\section{Irradiance and its Variability}

\subsection{Solar Radiation and Climate Experiment: SORCE}

The SORCE satellite was launched on 25 January 2003 and began routine operations by March 2003. The onboard instrumentation includes a Total Solar Irradiance Monitor (TIM), a Spectral Irradiance Monitor (SIM), Solar Stellar Irradiance Comparison Experiments (SOLSTICE), and an extreme ultraviolet (XUV) Photometer System (XPS). A comprehensive review of the instrumentation, its capabilities, and some of the new SORCE science results are provided in the special August 2005 edition of Solar Physics.

Space-based measurements of the total solar irradiance (TSI) began with the Earth Radiation Budget (ERB) experiment in 1979 and have continued uninterrupted for over a quarter of a century. Although there are substantial absolute differences between the measurements from different instruments, an excellent relative agreement has permitted the reconstruction of a composite TSI time series. For example, the ERB measurements were in the range of $1372-1375 \mathrm{~W} / \mathrm{m}^{2}$, while the Variability of Solar Irradiance and 
Gravity Oscillation (VIRGO) and Active Cavity Radiometer Irradiance Monitor (ACRIM III) data lie around $1365 \mathrm{~W} / \mathrm{m}^{2}$. The TIM measurements are even lower at $1361 \mathrm{~W} / \mathrm{m}^{2}$, and provide compelling evidence that the composite reconstruction, weighted heavily by the absolute value recorded by the VIRGO experiment, has overestimated the solar irradiance by $3-4 \%$, or $4-5 \mathrm{~W} / \mathrm{m}^{2}$. The sources of absolute offset between the different instruments are complex but hinge in large part on upon knowing the precise effective collecting aperture.

The implications of this reevaluation of the solar 'constant' for climate models is not presently known. Several prescriptions for the TSI variations with respect to the 16111998 'mean' of $1365.6 \mathrm{~W} / \mathrm{m}^{2}$ (Lean et al. 1995) are currently employed (Goosse et al. 2005; Jones \& Mann 2004) to account for the solar forcing in paleoclimate simulations spanning the last one or two millennia. However, it is important to note that the input TSI is routinely reduced by a factor of 4 to "account for the smaller fraction of the Earth's surface that is actually exposed to the Sun on average," and is then further reduced by $30 \%$ to account for the Earth's albedo (Jones \& Mann 2004). Taken together, this evidence suggests that the relative variations of the TSI are more important than its actual mean value.

The spectacular X17 flare of 28 October 2003 registered as a 267 ppm (parts per million) - or $0.34 \%$ - increase in the TSI recorded by TIM. The SORCE Team estimated that such a detection was consistent with the overall release of some $610^{32}$ ergs in electromagnetic radiation by the flare (Woods et al. 2004). The gigantic active region that spawned this flare contributed to the lowest TSI ever recorded on 29 October 2003, almost $3400 \mathrm{ppm}$ below the nominal TSI levels before the appearance of this active region. Interestingly the largest flare of the modern era (X28?) a few days later on 4 November 2003 did not produce as dramatic impacts on the TSI as its X17 predecessor, perhaps because of its orientation and its location nearer to the solar limb.

Another unexpected finding of the SORCE mission concerns the center-to-limb variation (CLV) of active region irradiance in the infrared (Fontenla et al. 2004). At visible wavelengths, it is well known that active regions contribute a net reduction in irradiance due to the presence of dark sunspots at disk center. At the limb, however, the foreshortening of the sunspot umbra no longer competes with the excess emission from the bright surrounding faculae, and so a net increase in irradiance results. Conventional wisdom held that this CLV behavior did not apply to the infrared, but SIM observations between 1.2-3 $\mu \mathrm{m}$, which includes the solar opacity minimum near $1.63 \mu \mathrm{m}$, show that the infrared exhibits the same CLV as the visible, only at a somewhat (50\%) reduced level. This result will require the revision of some of the semi-empirical models currently used to synthesize the solar spectrum (Fontenla et al. 1999).

\subsection{Modeling Facular Contrast}

Three separate groups have independently converged on a comprehensive and physically satisfying understanding of the center-to-limb appearance of solar faculae (Keller et al. 2004; Carlsson et al. 2004; Shelyag et al. 2004; Steiner 2005). These detailed numerical simulations were inspired by the superb observations recently obtained at the Swedish Vacuum Tower Telescope on La Palma (Lites et al. 2004). The essential explanation is basically the "bright wall" effect of the evacuated magnetic flux concentrations originally proposed by Spruit $(1976,1977)$, but with some additional wrinkles. "The brightness enhancement of the facula originates mainly from a thin layer near the limbward interface between the magnetic flux concentration and the hot non-magnetic granule. The intensity of the dark lane is formed in the relatively cool regions above the centerward granule and inside the flux concentration" (Keller et al. 2004). Carlsson et al. (2004) focused 
their attention on synthesis of the G-band $(430.68 \mathrm{~nm})$, which is often employed as a proxy for small-scale magnetic flux concentrations. They noted that the optical depth unity surface is very corrugated and drops into magnetic valleys where the temperature is greater. The reduced density within these valleys tends to destroy the $\mathrm{CH}$ molecules and further reduces the opacity in the G-band relative to the cooler and denser surrounding quiet Sun.

\subsection{Revised Solar Abundances of $C$ and $O$}

A series of papers by Asplund and collaborators (Asplund 2000; Asplund et al. 2004; Asplund 2004; Asplund et al. 2005) have led to significant revisions in solar photospheric abundances of carbon and oxygen. These findings are based on a complete analysis of a wide variety of spectral diagnostics associated with molecular electronic and vibrationrotation transitions as well as forbidden lines. The critical ingredients are a sophisticated three-dimensional hydrodynamical model of solar granulation coupled with detailed nonLTE synthesis of the emitted radiation. For both carbon and oxygen, the new abundances are decreased by almost a factor of 2 . This brings the photospheric $\mathrm{C} / \mathrm{H}$ and $\mathrm{O} / \mathrm{H}$ ratios into better agreement with abundance estimates obtained from the solar wind and solar flares, as well as neighboring B stars. There are additional implications for $\mathrm{Ne}$ and $\mathrm{Ar}$ abundances. As these elements to do not produce photospheric absorption features, their solar abundances must be inferred from solar wind and solar energetic particle events where their ratio relative to oxygen is readily determined. As neither Ne or Ar are expected to be susceptible to fractionation associated with the First Ionization Potential (FIP) effect, the factor of 2 reduction for $\mathrm{O}$ and $\mathrm{C}$ also applies to $\mathrm{Ne}$ and Ar.

Taken together, these effects lead to a significant reduction in the solar metallicity to $\mathrm{Z}=0.0126$. The effect is sufficiently great so as to introduce obvious disagreement between the standard solar model based on these new abundances and the seismic models derived from helioseismology (Antia \& Basu 2005; Bahcall et al. 2005).

\section{Helioseismology}

\subsection{Variations in Zonal and Meridional Flows}

Extended time series of Dopplergrams from the Michelson Doppler Imager (MDI) on board the Solar Heliospheric Observatory (SOHO) satellite, and the ground-based Global Oscillations Network Group (GONG) network are now available. Changes in solar dynamics and structure are manifest in a general increase in the frequencies of the solar acoustic oscillations with increasing magnetic activity. Detailed inversions that exploit differential frequency shifts between modes with different eigenfunctions are used to identify the underlying dynamic changes. These changes are generally restricted to the upper layers of the solar convection zone (SCZ).

Bands of alternating faster and slower zonal flow, the so-called "torsional oscillations" are observed to propagate both toward the poles and the equator from latitudes around $\pm 45^{\circ}-50^{\circ}$ (Basu \& Antia 2003). At a fixed low latitude there is strong evidence that the bands propagate upward in radius from the base of the SCZ. At a fixed high latitude, there is an opposite sense of downward radial propagation, again reaching all the way to the base of the SCZ. Relative to the surface differential rotation (Snodgrass 1984), the zonal flow anomalies are on the order of several meters per second throughout the entire convection zone. It is important to note that the magnetic activity belts are found to lie just to the poleward side of the bands of faster zonal flow (Zhao \& Kosovichev 2004). 
In a closely related study, Zhao et al. (2004) found that the residual (excess) rotation rate of relatively weak magnetic features (field strengths at or below $600 \mathrm{G}$ ) is highly correlated with their intrinsic field strength. The correlation is linear with a $300 \mathrm{G}$ magnetic flux concentration rotating about $80 \mathrm{~m} / \mathrm{s}$ faster than the mean background material. If this excess reflects an anchoring of the flux tube at depth below the surface, then given the inferred profile of the near surface shear, $600 \mathrm{G}$ elements would be rooted at approximately $0.95 \mathrm{R}_{\odot}$. Interestingly, this study revealed a clear asymmetry between leader and follower polarities in the sense that for the same magnetic field strength, the following polarity flux tubes had an excess rotation rate nearly a factor of two larger than their leader polarity counterparts.

The putative existence of an $\approx 1.5$ yr oscillation in the zonal flow of the tachocline still remains controversial. Some groups detect the oscillation in their data (Howe et al. 2000; Schou et al. 2002), while others do not (Basu \& Antia 2003).

The equator-to-pole quasi-steady surface meridional flow of some $20 \mathrm{~m} / \mathrm{s}$ also exhibits fluctuations that again seem to be tied to solar magnetic activity. There is a tendency for the strength of this flow to be somewhat suppressed when the Sun is magnetically active (Basu \& Antia 2003). Likewise, individual active regions invariably show a localized inward converging meridional flow superimposed upon the general background two-cell (distinct northern and southern hemisphere cells) pattern (Zhao \& Kosovichev 2004).

Significant asymmetries are generally present between the meridional near surface flows inferred for the northern and southern hemispheres. Indeed, between 1995 and 2002, Basu \& Antia (2003) find a steady north wind (i.e. southward flow) of a couple of meters per second is present at the solar equator at all times.

Neither the general background return pole-to-equator flow, nor the diverging meridional flow in the deep roots of the active region have been detected in the first $18 \mathrm{Mm}$ below the solar surface.

\subsection{Variations in Radius, Turbulence and Topography}

Although both p-mode and f-mode frequencies increase with solar magnetic activity, they exhibit opposite trends of frequency shift when viewed as a function of frequency, once the dominant contribution of the mode mass is removed. Dziembowski \& Goode (2004, $2005)$ point out that high frequency p-modes suffer greater shifts than their low frequency counterparts (in the $2-5 \mathrm{mHz}$ range), while low frequency f-modes are shifted more than their high frequency counterparts (in the $1.2-1.7 \mathrm{mHz}$ range).

These opposing trends, when combined with the powerful variational principles developed by Lynden-Bell \& Ostriker (1967), suggest that at solar maximum the increased magnetic flux in the near-surface layers leads to a $\approx 2 \%$ decrease in the strength of the turbulent velocities. This in turn acts to decrease the ambient surface temperature (by about $8 \mathrm{~K}$ ) and reduce the solar radius. However, as Spruit (2000) noted, the slight decrease in temperature is more than compensated by the magnetically-mediated corrugation of the optical depth unity surface, and so the smaller, cooler, more active Sun has an enhanced irradiance.

\subsection{Active Regions and Solar Subsurface Weather}

The local influence of active regions - specifically, their entrained magnetic fields - upon the solar acoustic oscillations is well known and is also problematic for efforts to deduce structure and dynamics under active regions. The most familiar example is the "absorption" of p-modes by sunspots first reported by Braun, Duvall \& LaBonte (1987). Recently, Cally, Crouch \& Braun (2003) have quantitatively confirmed Spruit's (1991) conjecture that the "absorption" is in fact a mode conversion process, where some of the 
energy of the incident p-modes is apportioned to MHD waves that propagate away from the solar surface along the magnetic lines of force. This process also endows the scattered p-modes with a phase shift. Using an empirical model that takes careful account of the inclination of the magnetic field lines with respect to the vertical, Cally et al. (2003) have reproduced Braun's (1995) observations of absorption and phase shifts as a function of incident p-mode parameters in exquisite detail.

Local helioseismology employs several techniques besides the Hankel analysis approach pioneered by Braun et al. (1987), including time-distance (cross correlations of acoustic surface fields), ring diagram (construction of local $k$ - $\omega$ diagrams), and phase sensitive holography (forward and backward propagation of acoustic surface fields to and from focal points) analyses. Unlike the Hankel approach, these techniques invariably make use of the oscillatory signals within the active regions. One of the attendant complications discovered in holography is the imprint of spurious surface phase shifts caused by the direct influence of the magnetic fields on the oscillations, commonly referred to as the acoustic "shower glass effect" (Lindsey \& Braun 2004; Lindsey \& Braun 2005a; Schunker et al. 2005). Inclined magnetic fields, like those routinely found in the penumbrae of sunspots, appear to be especially effective at producing significant phase shift, and attendant travel time anomalies.

These time-distance and ring diagram methods consistently associate complex subsurface flow patterns, "solar subsurface weather" (SSW), with active region complexes (Hindman et al. 2004). The derived horizontal flow patterns show fascinating variations in both space and time. As noted earlier, horizontal near surface flows tend to converge toward individual sunspots and active region complexes (Zhao \& Kosovichev 2004), which implies a general subsidence within these regions and a corresponding outflow at greater depths. The analogy with terrestrial weather, replete with traveling vortical structures reminiscent of high and low pressure areas, has motivated some recent efforts to actually frame the SSW problem in terms of quasi-geostrophic flow systems (Spruit 2003).

Some initial caution in over interpretation of SSW results is probably called for. The full influence of magnetic surface effects needs to be quantified, through forward modeling, and perhaps even "surgically" removed from the surface oscillation data where appropriate (Lindsey \& Braun 2005b). A detailed authoritative account of these highlights, and many other developments in local helioseismology, can be accessed in the forthcoming Living Review article by Gizon \& Birch (2005).

\section{Surface Magnetism}

\subsection{Sunspots}

The advent of new polarimeters, specially in the infrared (IR) region, has provided important progress in the area of sunspot magnetism. Using the Spectropolarimeter for Infrared and Optical Regions (SPINOR), Socas-Navarro (2005) has analyzed full Stokes profiles of the chromospheric lines of Ca II at 849.8 and $854.2 \mathrm{~nm}$. Non-LTE inversions of this data permit the retrieval of the full atmospheric parameters (magnetic field, temperature, etc.) from the photosphere to the chromosphere, spanning a range of heights as large as $1600 \mathrm{~km}$ above the photosphere. This analysis shows that, even for the case of sunspot umbrae, one finds configurations that depart significantly from a potential state displaying oppositely twisted umbral magnetic cores. It is clear that, with this technique, meaningful derivations of the full electric current vector will soon be available (not just the z-component). 
The existence of twist in sunspots, largely unexpected, can be related to another surprising discovery that has passed relatively unnoticed and whose relevance will become evident in the coming years. We refer to the existence of rotating sunspots as observed by the Transition Region And Coronal Explorer (TRACE, Brown et al. 2003). Uninterrupted animations made at various TRACE wavelengths have shown how some sunspots are seen to rotate at rates of 40 degrees per day. The rotation is seen more evidently in the fanning coronal loops. These rotations last for periods of several days. All these observations show that, in the near future, quantifying the twist of the flux ropes produced by the dynamo (Charbonneau 2005) will be a real possibility.

After the initial discovery of dark core penumbral filaments (Scharmer et al. 2002), more evidence of their ubiquitous existence has now been gathered (Rouppe van der Voort et al. 2004; Rimmele 2004; Sütterlin et al. 2004; Puschmann \& Kneer 2005; Denker et al. 2005). The presence of these dark cores is thought to be related to the horizontal magnetic component that is normally used in the context of spectropolarimetric observations; but a definite model has not been produced yet. This model with horizontal flux tubes carrying the Evershed flow embedded in a more vertical background is broadly used to explain a number of observations. IR observations by Bellot Rubio et al. (2004) have been interpreted in this context deriving properties of the two components (horizontal and vertical) as a function of radial distance within the spot. The smaller range of formation heights of the IR lines compared to commonly used visible lines, allows a clear separation between the two components. Models that use a magnetic equilibrium configuration with an embedded tube are being used (Borrero et al. 2005) to reproduce the Stokes profiles. The resolution of these spectroscopic data is seldom better than $1 \operatorname{arcsec}(750 \mathrm{~km})$. But imaging magnetograms using Adaptive Optics techniques provide snapshots that occasionally reach ten times better resolution, 0.1 arcsec or $75 \mathrm{~km}$ on the Sun (see Langhans et al. 2005). At this resolution, coherent structures that extend radially over the entire penumbra are observed. The fluctuations observed in the inclination reach up to 45 degrees in nearby pixels. Dark core penumbral filaments correlate one-to-one with the horizontal component carrying the Evershed flow. These results show how enormous has been our increased understanding of the magnetic configuration of the penumbra in recent years. How exactly such a configuration forms and links to the generation of the Evershed flow and to the problem of energy transport in the penumbra remains controversial, though (Schlichenmaier \& Solanki 2003; Weiss et al. 2004; Thomas 2005). The structuring of the penumbra in two magnetic components differently related to the Evershed flow has been used by Sainz Dalda \& Martínez Pillet (2005) to explain bipolar Moving Magnetic Features as the evolution of this flow once it leaves the spot.

\subsection{Quiet Sun Magnetism}

The magnetism of the quiet Sun has remained a lively topic. Progress has been made in three areas: Stokes signals due to the Zeeman effect in the visible, in the IR and Hanle signals in the visible. Notably, each one seems to provide a different answer to what is the magnetic state of the so-called internetwork regions. No doubt part of the problem resides in the definition of what is an internetwork area (Lites \& Socas-Navarro 2004). High resolution ( 0.5 arcsec) magnetograms in the visible, after speckle reconstruction, provide an average unsigned flux in the quiet sun as large as $20 \mathrm{G}$ (Domínguez-Cerdeña et al. 2003). But observations made with the Diffraction Limited Stokes Polarimeter (DLSP), achieving 0.6 arcsec resolution, infer a value of only $8 \mathrm{G}$ (Lites \& Socas-Navarro 2004). Not only the mean fluxes are controversial, but so are the intrinsic field strengths. Most of the detected signals in the visible correspond to $\mathrm{kG}$ fields. In the IR, one finds mostly field strengths in the 200-600 G range (Khomenko et al. 2003). At 1 arcsec resolution the 
average net flux is also near $8 \mathrm{G}$, but this time is due to $50 \%$ of the area covered by weak fields instead of concentrated strong fields (as is seen in the visible). It is clear that the two spectral regions are sensitive to different fields and that a Probability Distribution Function (PDF) is needed to describe the magnetic fields found in the internetwork. This is true everywhere in the Sun, but seems to be particularly important for internetwork regions. While no consensus on the shape of this PDF exits, it seems clear that visible lines will provide the high field strength wings of the PDF, IR lines the intermediate region and Hanle signals the region at and below 100 G (Socas-Navarro \& Sánchez Almeida 2003; Trujillo Bueno et al. 2004; Sánchez Almeida 2005). It is important to remark that even if Hanle signals are more sensitive to the low field strength range of the PDF, reservoirs of unsigned flux as large as $100 \mathrm{G}$ are routinely observed (Trujillo Bueno et al. 2004).

The above studies rely heavily on spectropolarimetric data, which provides the most complete information. The penalty is the relatively poor spatial resolution achievable. Imaging with the Swedish Solar Telescope (SST) at La Palma, has provided a new view of the structuring of small scale magnetic fields. Berger et al. (2004) have obtained G-band images and a number of coeval magnetograms at Fe I $630.2 \mathrm{~nm}$ in a relatively strong plage region. At 0.1 arcsec resolution, high flux concentrations show ribbon structures in G-band frames. These ribbons do not resolve themselves into flux tube-like structures, but more into flux sheets as seen in MHD simulations. Isolated dot-like structures are found only in lower flux density areas. The interesting point is that, in these structures, often one finds magnetogram signals (with 0.2 arcsec resolution) in the $\mathrm{kG}$ regime (always relying on complex calibrations, however). If these $\mathrm{kG}$ signals can be trusted, and given the fact that we know these are typical intrinsic field strength values, one should conclude that the filling factor is close to unity. That is, at these resolutions magnetic fields would be filling the whole resolution element. It is crucial to confirm in future work that $\mathrm{kG}$ signals are routinely obtained at these spatial resolutions.

At higher spatial scales and flux concentrations, ephemeral regions have proven to be a key ingredient of network magnetism. Hagenaar et al. (2003) have found that the ephemeral region population is a continuation of the active region spectrum. The whole spectrum is a smooth decrease function that spans 4 orders of magnitude in flux and 8 orders of magnitude in frequency. The low end of the ephemeral regions spectrum would be in antiphase with the solar cycle at a much reduced amplitude (variation from minimum to maximum of 1.5). For these reasons, these authors propose that this range of ephemeral-regions $\left(10^{19-20} \mathrm{Mx}\right)$ would be generated by a surface, small-scale, dynamo mechanism (see $\$ 6.2$ ). This would fit with the findings of a cycle independent (within a factor 2) number of X-ray bright points, as found by Hara \& Nakakubo-Morimoto (2003).

\section{Structure of the Quiet Sun Chromosphere}

The debate on the thermal structuring of the chromosphere continues. Whereas most of the previous discussion has concentrated on whether the mean chromosphere is hot or cold and the magnitude of the temporal fluctuations, new results put more emphasis on spatial thermal structuring. Three-dimensional simulations by Wedemeyer et al. (2004), extending from the convective zone into the middle chromosphere, result in a spatially bifurcated chromospheric temperature. Hot and cold regions coexists at the same time. Convective motions generate sound waves, mostly at intergranular, regions that propagate upwards and steepen into shocks. A variety of sound waves and shocks exists at each height. The local interactions among them determine which parts of the atmosphere are cold and which are hot. While this high degree of structuring is not unexpected, the details still remain controversial. The interaction of shock waves and how they merge 
depends on the assumed geometry and, if incorrectly described, may lead to an artificial destruction of high-frequency acoustic wave power in the simulations (Ulmschneider et al. 2005). An effort should be made to undertake correct modeling of shock wave interactions because, as shown by Fossum \& Carlsson (2005), the power obtained in simulations of the intensity fluctuations observed by TRACE in the high frequency range is not enough for acoustic heating of these layers.

Advances in the field of chromospheric magnetism have relied considerably on the use of the He I $1083 \mathrm{~nm}$ line. Progress in understanding the formation of this line and its coupling to coronal radiation, which complicates its diagnostic, has been made by Mauas et al. (2005). But a full treatment of the line formation in the presence of magnetic fields including both the Zeeman and Hanle effects is still not available. Nevertheless, simple radiative transfer models, like the Milne-Eddington approximation, have allowed meaningful retrieval of the vector magnetic field in the chromosphere with unprecedented accuracy (Lagg et al. 2004). In this way, the detection of tangential discontinuities of the magnetic field in the chromosphere has been possible (Solanki et al. 2003). Additionally, detailed observation and analysis of the He line, but now including atomic polarization and Hanle effect diagnostics, has also provided, for the first time, the vector magnetic field in chromospheric spicules. Trujillo Bueno et al. (2005) have observed with the Tenerife Infrared Polarimeter (TIP) instrument a field strength of the order of $10 \mathrm{G}$ and an inclination with respect to the vertical of $35^{\circ}$. This orientation fits rather nicely with the inclined field lines that have been proposed as a mechanism for p-mode leaking into the chromosphere to explain the formation of spicules (De Pontieu et al. 2004).

\section{Solar Instrumentation}

The SST (1m aperture) has entered into full operation. The increase of a factor 2 in the aperture has provided a wealth of new results that illustrate the tremendous benefits from such an increase. The old aperture had a resolution limit of, typically, $150 \mathrm{~km}$ over the solar surface. Since operation, the SST has shown that resolving $75 \mathrm{~km}$ provides important benefits beyond what a mere factor 2 would suggest. The penumbra is consistently seen structured into filaments displaying dark cores and bright rims that evolve synchronously (Scharmer et al. 2002; see §3.1)). Facular regions show ubiquitous ribbon like structures in G-band images with $\mathrm{kG}$ fields in magnetogram signals (Berger et al. 2004; see §3.2). Observations of facular and network regions near the limb show three dimensional structures, making evident the Wilson depression in pores and facular brightenings projected on the granulation closer to the limb but not on the other side (Lites et al. 2004; see §1.2). Granules observed at this resolution show a 'hilly' morphology. The comparison of these observations with MHD simulations of the interaction of magnetic fields and solar convection shows a striking degree of similarity (Keller et al. 2004; Carlsson et al. 2004; see $§ 1.2$ ). All this evidence points to the benefits of resolving fundamental length scales of the solar surface like the pressure scale height and the photon mean free path. These results refer to imaging in continuum bands with sufficient photons to allow the use of millisecond exposure times (but that still need further image reconstruction). These resolutions are far from achievable for spectropolarimetric observations which should be the goal for the coming years in solar instrumentation.

Four grating spectropolarimeters are already available integrated with adaptive optic systems. The Tenerife Infrared Polarimeter (TIP) has been integrated with the Kiepenheuer Adaptive Optic System (KAOS, von der Lühe et al. 2003) for routine operations. The combination TIP-KAOS has provided IR spectropolarimetric maps (slit-scanning) at resolutions of 1 arcsec, and occasionally slightly better. The Polarization Littrow 
Spectrograph (POLIS) visible spectropolarimeter (Schmidt et al. 2003) can be coupled with TIP (and also KAOS) to provide simultaneous wavelength coverage from the $630 \mathrm{~nm}$ region up to $2 \mu \mathrm{m}$. At the Dunn Solar Telescope in Sac Peak, the Diffraction Limited Stokes Polarimeter (DLSP, Sankarasubramanian et al. 2004) and the Spectropolarimeter for the Infrared and Optical Regions (SPINOR, Socas-Navarro et al. 2005b) have enter into operation. It is clear that the integration of Adaptive Optics (AO) into all these instruments will significantly improve the spatial resolution achieved by spectropolarimeters. However a note of caution must be given. Whenever a slit scanning map obtained in the visible is compared with a simultaneous map made in the IR, the latter has better image quality (as it normally occurs with the TIP-POLIS combination). This readily shows that the data are still seeing limited and that AO systems are still far from providing diffraction limited data when exposure times of several seconds are used. Diffraction limited spectropolarimetry is still not guaranteed through current AO systems. In this sense, the data to be obtained by the spectropolarimeter that will fly on the Japanese-led Solar-B mission (launch in 2006) will be unique, with no possible comparison with data from similar ground instruments.

The Advanced Technology Solar Telescope (ATST, Keil et al. 2004) led by the National Solar Observatory has progressed steadily. Major steps have been the project reviews at conceptual and preliminary levels and the site selection process. Haleakala (Hawaii) has been selected as the primary candidate for the ATST, while La Palma (Canary Islands) and Big Bear (California) remain as back-up sites. Currently, first light is planned for 2013. The Synoptic Optical Long-term Investigation of the Sun (SOLIS, Harvey et al. 2004) facility has entered full operation. Photospheric vector magnetograms are being obtained on a daily basis, together with longitudinal chromospheric full-disk images. The sensitivity of the data is as high as $1 \mathrm{G}$, which represents an order of magnitude better than previous facilities. Magnetograms and He I $1083 \mathrm{~nm}$ filtergrams are available on the web. Both the addition of vector capabilities at photospheric levels and the chromospheric data will enormously improve the capabilities of synoptic research of the solar magnetic cycle.

\section{Dynamo and Magnetoconvection}

\subsection{Flux Transport Dynamo}

A particularly robust empirical model of the solar magnetic dynamo has been constructed on the framework of the Babcock-Leighton approach (Babcock 1961; Leighton 1969). The critical additional ingredient is the incorporation of the quasi-steady meridional flow discussed in $§ 2.1$ above (Dikpati et al. 2004; Charbonneau 2005; Dikpati 2005). The posited closure of the meridional convective cell near the base of the SCZ creates a "conveyor belt," that is capable of recycling the surface magnetic fields back into the tachocline where they can be reprocessed by differential rotation and cyclonic motions. As the return flow at depth is quite weak, a complete circuit around the cell can be in the range of $30-60 \mathrm{yrs}$, although the more important time scale is the 10-16 yr time scale for low latitude surface flux to be transported poleward and submerged into the high latitude tachocline.

The model does an outstanding job of reproducing nearly every attribute of the observed solar magnetic cycle. More important, however, are its powerful predictive capabilities. This is possible because the "conveyor belt" transport of magnetic flux endows the dynamo with a memory of previous cycles. The variable speed of the meridional circulation also impacts the timing of solar minimum and cycle length. Based on the 
deceleration of the meridional flow with decreasing magnetic activity observed during the declining phase of cycle 23, Dikpati and her collaborators have predicted that the onset of cycle 24 will commence in late 2007 or early 2008.

\subsection{Dynamos in the Small}

The accumulating evidence supports the concept that two rather different sorts of dynamos are operating concurrently within the Sun: one is the familiar large-scale dynamo discussed in the previous section, the other operates on the small and fast granular flow scale (Cattaneo 1999; Cattaneo et al. 2003; Sánchez Almeida et al. 2003; Vögler et al. 2005). Unlike the large-scale dynamo which knows about the solar rotation and produces coherent regions of unipolar flux (active regions), the small-scale dynamo produces lots of weak and highly intermittent magnetic field, but has no particular preference for one polarity over the other. Fields created by the small-scale dynamo are preferentially swept into the intergranular lanes where they form the magnetic network, the roots of the magnetic carpet, and the G-band bright points and faculae described in $\S 1.2$ above.

The small-scale dynamo obviously feeds off the erupting magnetic flux created by the large-scale dynamo and its activity can be inhibited when the Lorentz forces associated with the emerging magnetic flux become too large (Cattaneo et al. 2003; Vögler et al. 2005). This situation is quite reminiscent of the changes in solar surface structure invoked by Dziembowski \& Goode $(2004,2005)$ to explain the variability of the p-mode and f-mode frequency shifts (see $\S 2.4$ ).

\subsection{Solar Convection Zone (SCZ) Dynamics}

One of the truly spectacular recent advances in studies of the SCZ has been the work of the group based at the University of Colorado (Brun et al. 2004). Using an anelastic formulation of the MHD equations ab initio three-dimensional simulations of the solar envelope (roughly $0.62 \mathrm{R}_{\odot} \leqslant r \leqslant 0.98 \mathrm{R}_{\odot}$ ) have been carried out at the highest resolution presently permitted by computing capacity. The initial findings are particularly encouraging, and include realistic differential rotation profiles, meridional flows (albeit more complex than the one-cell per hemisphere pattern that appears to pertain to the Sun), and sustained dynamo action (although not characterized by the large scale regularity present in the Sun).

As Miesch (2005) notes, "there is a marked contrast between the convective structure at low and high latitude. Near the equator, the convection is dominated by extended downflow lanes oriented north-south which propagate in longitude faster than the local differential rotation. Near the poles the convection patterns are more isotropic and homogeneous and the characteristic spatial scales are somewhat smaller."

\section{Steady Coronal Structure}

\subsection{Measurement of the Coronal Magnetic Field}

A recent breakthrough of unprecedented proportion is the measurement of coronal vector magnetic fields with sensible spatial and temporal resolution (Lin et al. 2004; Penn et al. 2004). Critical advances in modern infrared array detectors coupled with a complete quantum mechanical theory of polarized transfer in the presence of atomic alignment (Casini 2002) have made this possible. The forbidden infrared emission line of Fe XIII at $1075 \mathrm{~nm}$ (Landé g-factor of 1.5) is particularly well-suited to this task (Judge et al. 2001).

At a height of $0.1-0.15 \mathrm{R}_{\odot}$ above the limb, the typical field strengths are on the order of $4 \mathrm{G}$ near the apex of active region loops. The projection of the magnetic field in the 
plane of the sky is obviously influenced by the topology of the emission features (as recorded concurrently in the Fe XV $28.4 \mathrm{~nm}$ line intensity), but is not necessarily aligned with these structures. One potential cause for this misalignment is the Van Vleck effect, which rotates the polarization perpendicular to the actual magnetic field when the local field inclination in the scattering region is in the neighborhood of $55^{\circ}$.

\subsection{Acceleration of the Solar Wind}

Measurements of Si II $(153.3 \mathrm{~nm})$, C IV $(154.8 \mathrm{~nm})$ and Ne VIII $(77.0 \mathrm{~nm})$ line profiles obtained with the Solar Ultraviolet Measurements of Emitted Radiation (SUMER) instrument on board the Solar and Heliospheric Observatory (SOHO) satellite have established that the solar wind begins flowing in magnetic funnels between the altitudes of $5 \mathrm{Mm}$ and $20 \mathrm{Mm}$ above the chromosphere (Tu et al. 2005; Wiegelmann et al. 2005). The extrapolation of coeval Michelson Doppler Imager (MDI) photospheric magnetograms obtained within a coronal hole to coronal altitudes permits the unambiguous identification of closed magnetic loops (with apex altitudes at or below $5 \mathrm{Mm}$ ) and open magnetic field funnels. When maps of the intensity and Doppler velocities of the ultraviolet emission lines are inserted at the appropriate altitudes in the extrapolated magnetic fields a convincing correlation is found between Ne VIII upflows (of order $10 \mathrm{~km} / \mathrm{s}$ ) and the open magnetic funnels.

At lower altitudes where the funnels begin to constrict the C IV emission line forms from plasma that is largely at rest. Still deeper in the funnel where the Si II originates, $2 \mathrm{~km} / \mathrm{s}$ downflows are observed. This progression prompts Tu et al. (2005) to speculate that closed loops within the supergranule flow fields "may finally move to a funnel region and undergo reconnection with existing open fields. Thereby, plasma that is previously confined in the loops is released, which may lead to both upflow and downflows" as observed.

\subsection{Magnetic Evolution at All Scales}

Ab initio numerical calculations of the braiding of small scale magnetic fields have made a dramatic step forward in reproducing the dynamic character of the coronal plasma, including the requisite mechanical heating through magnetic dissipation to temperatures in excess of $10^{6} \mathrm{~K}$ (Gudiksen \& Nordlund 2005a; Gudiksen \& Nordlund 2005b; Peter et al. 2004). The MHD simulations, set in a $60 \mathrm{Mm} \times 60 \mathrm{Mm} \times 37 \mathrm{Mm}$ domain, essentially validate the basic magnetic (i.e., nanoflare) coronal heating mechanism that has been proposed and advocated by Parker. At the same time similar ab initio calculations pertaining to the solar chromosphere have further ruled out the possibility that high frequency $(10-50 \mathrm{mHz})$ acoustic waves may be responsible for heating the underlying chromosphere (Fossum \& Carlsson 2005).

These coronal calculations extend the concept, originally developed in a chromospheric context by Carlsson \& Stein (1997), that the quasi-steady solar atmosphere in fact represents a radiation-weighted coarse-grained average over a whole spectrum of highly localized and extremely nonlinear small-scale dynamics. A particularly beautiful aspect of the Gudiksen \& Nordlund (2005b) simulation is the ubiquitous appearance of bright emission loops in C IV (154.8 $\mathrm{nm})$ and $\mathrm{Mg} \mathrm{X}(62.5 \mathrm{~nm})$, after the simulations are subjected to a post-processing spectral synthesis (Peter et al. 2004). Even more dramatic is the outstanding agreement between the differential emission measure (DEM) versus line formation temperature derived from the simulations with those routinely observed in the solar atmosphere. A particularly intriguing, and perhaps prophetic, finding is that the simulated emission loops do not necessarily follow magnetic flux bundles. 
At the other extreme in temporal and spatial scales, Knaack and collaborators (Knaack \& Stenflo 2005; Knaack et al. 2005) have recently computed time series of spherical harmonic coefficients from 38 years of Mt. Wilson and Kitt Peak magnetograms. This exhaustive study was based on over $610^{4}$ individual magnetograms spanning the period between September 1966 and September 2004. The 22 yr magnetic Hale cycle is the strongest feature in the power spectra, followed by its second (11 yr) and third (7 yr) harmonics. The novel use of wavelet analysis techniques further reveals the intermittent presence of oscillations with periods of $2.1-2.5 \mathrm{yr}, 1.5-1.8 \mathrm{yr}$, and $1.2-1.4 \mathrm{yr}$. The latter period has been reported in other time series related to solar cycle activity, for example, the controversial variability of the tachocline (see $\S 2.1$ ).

\section{References}

Antia, H.M., Basu, S. 2005, Astrophys. J. 620, L129

Asplund, M. 2000, Astron. Astrophys. 359, 755

Asplund, M., Grevesse, N., Sauval, A.J., Allende Pietro, C., Kiselman, D. 2004, Astron. Astrophys. 417,751

Asplund, M. 2004, Astron. Astrophys. 417, 769

Asplund, M., Grevesse, N., Sauval, J., Allende Pietro, C, Blomme, R. 2005, Astron. Astrophys. 431,693

Babcock, H.W. 1961, Astrophys. J. 133, 572

Bahcall, J.N., Basu, S., Pinsonneault, M., Serenelli, A.M. 2005, Astrophys. J. 618, 1049

Basu, S., Antia, H.M. 2003, Astrophys. J. 585, 553

Bellot Rubio, L.R., Balthasar, H., Collados, M. 2004, Astron. Astrophys. 427, 319

Berger, T.E., Rouppe van der Voort, L.H.M., Löfdahl, M.G., Carlsson, M., Fossum, A., Hansteen, V.H., Marthinussen, E., Title, A., Scharmer, G. 2004, Astron. Astrophys. 428, 613

Borrero, J.M., Lagg, A., Solanki, S.K., Collados, M. 2005, Astron. Astrophys. 436, 333

Braun, D.C. 1995, Astrophys. J. 451, 859

Braun, D.C., Duvall, T.L., Jr., LaBonte, B.J. 1987, Astrophys. J. 319, L27

Brown, D.S., Nightingale, R.W., Alexander, D., Schrijver, C.J., Metcalf, T.R., Shine, R.A., Title, A.M., Wolfson, C.J. 2003, Sol. Phys. 216, 79

Brun, A.S., Miesch, M.S., Toomre, J. 2004, Astrophys. J. 614, 1073

Cally, P.S., Crouch, A.D., Braun, D.C. 2003, MNRAS 346, 381

Carlsson, M., Stein, R.F. 1997, Astrophys. J. 481, 500

Carlsson, M., Stein, R.F., Nordlund, Å., Scharmer, G.B. 2004, Astrophys. J. 610, L140

Casini, R. 2002, Astrophys. J. 568, 1056

Cattaneo, F. 1999, Astrophys. J. 515, L39

Cattaneo, F., Emonet, T., Weiss, N.O. 2003, Astrophys. J. 588, 1183

Charbonneau, P. 2005, Living Rev. Solar Phys. http://www.livingreviews.org/lrsp-2005-2

De Pontieu, B., Erdélyi, R., James, S.P. 2004, Nature 430, 536

Denker, C., Mascarinas, D., Xu, Y., Cao, W., Yang, G., Wang, H., Goode, P., Rimmele, T. 2005, Sol. Phys. 227, 217

Dikpati, M. 2005, Adv. Space Res. 35, 322

Dikpati, M., De Toma, G, Gilman, P.A., Arge, C., White, O.R. 2004, Astrophys. J. 601, 1136

Domínguez-Cerdeña, I., Sánchez Almeida, J., Kneer,F. 2003, Astron. Astrophys. 407, 741

Dziembowski, W.A., Goode, P.R. 2004, Astrophys. J. 600, 464

Dziembowski, W.A., Goode, P.R. 2005, Astrophys. J. 625, 548

Fontenla, J.M., White, O.R., Fox, P., Avrett, E.H., Kurucz, R.L. 1999, Astrophys. J. 518, 480

Fontenla, J.M., Harder, J., Rottman, G., Woods, T.N., Lawrence, G.M., Davis, S. 2004, Astrophys. J. 605, L55

Fossum, A., \& Carlsson, M. 2005, Nature 435, 919

Gizon, L, Birch, A.C. 2005, Living Rev. Sol. Phys. http://www.livingreviews.org/lrsp-2005-6

Goosse, H., Crowley, T.J., Ammann, C.M., Renssen, H., Driesschaert, E. 2005, Geophys. Res. Lett. 32, L06710, doi:10.1029/2005GL022368 
Gudiksen, B.V., Nordlund, A 2005a, Astrophys. J. 618, 1020

Gudiksen, B.V., Nordlund, Å 2005b, Astrophys. J. 618, 1031

Hagenaar, H.J., Schrijver, C.J., Title, A.M. 2003, Astrophys. J. 584, 1107

Hara, H., Nakakubo-Morimoto, K. 2003, Astrophys. J. 589, 1062

Harvey, J., Giampapa, M., Henney, C., Keller, C., Jones, H. 2004, AASM 204, 37.01

Hindman, B.W., Gizon, L., Duvall, T.L., Jr., Haber, D., Toomre, J. 2004, Astrophys. J. 613, 1253

Howe, R., Christensen-Dalsgaard, J., Hill, F., Komm, R.W., Larsen, R.M., Schou, J., Thompson, M.J., Toomre, J. 2000, Science 287, 2456

Jones, P.D., Mann, M.E. 2004, Rev. Geophys. 42, RG2002

Judge, P.G., Casini, R., Tomczyk, S., Edwards, D.P., Francis, E. 2001, NCAR Technical Note, TN446, NCAR, Boulder

Khomenko, E.V., Collados, M., Solanki, S.K., Lagg, A., Trujillo Bueno, J. 2003, Astron. Astrophys. 408,1115

Keil, S.L., Rimmele, T.R., Oschmann, J., Hubbard, R., Warner, M., Price, R., Dalrymple, N., the ATST team 2004, IAU Symp. 223, A.V. Stepanov, E.B. Benevolenskaya \& Kosovichev, A.G. (eds.), CUP, 581

Keller, C.U, Schüssler, M., Vögler, A., Zakharov, V. 2004, Astrophys. J. 607, L59

Knaack, R., Stenflo, J.O. 2005, Astron. Astrophys. 438, 349

Knaack, R., Stenflo, J.O., Berdyugina, S.V. 2005, Astron. Astrophys. 438, 1067

Lagg, A., Woch, J., Krupp, N., Solanki, S.K. 2004, Astron. Astrophys. 414, 1109

Langhans, K., Scharmer, G.B., Kiselman, D., Löfdahl, M.G., Berger, T. 2005, Astron. Astrophys. 436, 1087

Lean, J., Beer, J., Bradley, R. 1995, Geophys. Res. Lett. 22, 1591

Leighton, R.B. 1969, Astrophys. J. 156, 1

Lin, H., Kuhn, J.R., Coulter, R. 2004, Astrophys. J. 613, L177

Lindsey, C., Braun, D.C. 2004, Astrophys. J. Suppl. 155, 209

Lindsey, C., Braun, D.C. 2005a, Astrophys. J. 620, 117

Lindsey, C., Braun, D.C. 2005b, Astrophys J. 620, 1118

Lites, B.W., Socas-Navarro, H. 2004, Astrophys. J. 613, 600

Lites, B.W., Scharmer, G.B., Berger, T.E., Title, A.M. 2004, Sol. Phys. 221, 65

Lynden-Bell, D, Ostriker, J.P. 1967, MNRAS 136, 557

Mauas, P.J.D., Andretta, V., Falchi, A., Falciani, R., Teriaca, L., Cauzzi, G. 2005, Astrophys. J. 619,604

Miesch, M.S. 2005, Living Rev. Sol. Phys. http://www.livingreviews.org/lrsp-2005-1

Penn, M.J., Lin, H., Tomczyk, S., Elmore, D., Judge, P. 2004, Sol. Phys. 222, 61

Peter, H., Gudiksen, B.V., Nordlund, Å 2004, Astrophys. J. 617, L85

Puschmann, K.G. \& Kneer, F. 2005, Astron. Astrophys. 436, 373

Rouppe van der Voort, L.H.M., Löfdahl, M.G., Kiselman, D., Scharmer, G.B. 2004, Astron. Astrophys. 414, 717

Rimmele, T., 2004, Astrophys. J. 604, 906

Sainz Dalda, A., Martínez Pillet, V. 2005, Astrophys. J. 632, 1176

Sánchez Almeida, J. 2005, Astron. Astrophys. 438, 727

Sánchez Almeida, J., Emonet, T., Cattaneo, F. 2003, Astrophys. J. 585, 536

Sankarasubramanian, K., Rimmele, T.R., Lites, B.W. 2004, AAS Meeting 204, 20.06

Scharmer, G.B., Gudiksen, B.V., Kiselman, D., Löfdahl, M.G., Rouppe van der Voort, L.H.M. 2002, Nature 420, 6912, 151

Schlichenmaier, R., Solanki, S.K. 2003, Astron. Astrophys. 411, 257

Schmidt, W., Beck, C., Kentischer, T., Elmore, D., Lites, B.W. 2003, AN 324, 4, 300

Schunker, H., Braun, D.C., Cally, P.S., Lindsey, C. 2005, Astrophys. J. 621, L149

Shelyag, S., Schüssler, M., Solanki, S.K., Berdyugina, S.V., Vögler, A. 2004, Astron. Astrophys. 427,335

Snodgrass, H.B. 1984, Sol. Phys. 94, 13

Socas-Navarro, H., Sánchez Almeida, J. 2003, Astrophys. J. 593, 581

Socas-Navarro, H. 2005, Astrophys. J. 631, L167 
Socas-Navarro, H., Elmore, D., Pietarila, A., Darnell, A., Lites, B.W., Tomczyk, S., Hegwer, S. 2005, Sol. Phys. in press

Solanki, S.K., Lagg, A., Woch, J., Krupp, N., Collados, M. 2003, Nature 425, 6959, 692

Spruit, H.C. 1976, Sol. Phys. 50, 269

Spruit, H.C. 1977, Sol. Phys. 55, 3

Spruit, H.C. 1991, in Challenges to Theories of the Structure of Moderate Mass Stars. Lecture Notes in Physics, Vol. 388, Toomre, J, Gough, D.O., eds., Springer-Verlag, Berlin, 121

Spruit, H.C. 2000, Space. Sci. Rev. 94, 113

Spruit, H.C. 2003, Sol. Phys. 213, 1

Steiner, O. 2005, Astron. Astrophys. 430, 691

Sütterlin, P., Bellot Rubio, L., Schlichenmaier, R. 2004, Astron. Astrophys. 424, 1049

Thomas, J.H. 2005, Astron. Astrophys. 440, L29

Trujillo Bueno, J., Merenda, L., Centemo, R., Collados, M., Landi Degl'Innocenti, E. 2005, Astrophys. J. 619, L191

Trujillo Bueno, J., Shchukina, N., Asensio Ramos, A. 2004, Nature 430, 6997, 326

Tu, C.-Y., Zhou, C., Marsch, E., Xia, L.-D., Zhao, L., Wang, J.-X., Wilhelm, K. 2005, Science 308,519

Ulmschneider, P., Rammacher, W., Musielak, Z.E., Kalkofen, W. 2005, Astrophys. J. 631, L155

Vögler, A., Shelyag, S., Schüssler, M., Cattaneo, F., Emonet, T., Linde, T. 2005, Astron. Astrophys. 429,335

von der Lühe, 0., Soltau, D., Berkefeld, T., Schelenz, T. 2003, SPIE 4853, 187

Wedemeyer, S., Freytag, B., Steffen, M., Ludwig, H.G., Holweger, H. 2003, Astron. Astrophys. 414,1121

Weiss, N.O., Thomas, J.H., Brummell, N.H., Tobias, S.M. 2004, Astrophys. J. 600, 1073

Wiegelmann, T., Xia, L.-D., March, E. 2005, Astron. Astrophys. 432, L1

Woods, T.N., Eparvier, F.G., Fontenla, J., Harder, J., Kopp, G., McClintock, W.E. Rottman, G., Smiley, B., Snow, M. 2004, Geophys. Res. Lett. 31, CiteID L10803, doi:10.1029/2004GL019571

Zhao, J., Kosovichev, A.G. 2004, Astrophys. J. 603, 776

Zhao, J., Kosovichev, A.G., Duvall, T.L., Jr. 2004, Astrophys. J. 607, L135 